S.-S. Lee

Nagoya Math. J.

Vol. 91 (1983), 173-184

\title{
NON-LINEAR PREDICTION PROBLEMS FOR ORNSTEIN-UHLENBECK PROCESS
}

\author{
SHEU-SAN LEE
}

\section{§. Introduction}

We shall discuss in this paper some problems in non-linear prediction theory. An Ornstein-Uhlenbeck process $\{U(t)\}$ is taken to be a basic process, and we shall deal with stochastic processes $X(t)$ that are transformed by functions $f$ satisfying certain condition. Actually, observed processes are expressed in the form $X(t)=f(U(t))$. Our main problem is to obtain the best non-linear predictor $\hat{X}(t, \tau)$ for $X(t+\tau), \tau>0$, assuming that $X(s), s \leq t$, are observed. The predictor is therefore a non-linear functional of the values $X(s), s \leq t$.

Non-linear prediction theory that discusses how to obtain such nonlinear predictors has been considered in various situations. For instance, I. I. Gihman and A. V. Skorohod (cf. [1], §8, Chapter IV, Vol. I) have considered optimum mean square predictor of $X(t+\tau), \tau>0$, assuming that the basic process $V(s), s \leq t$, itself is observed. As is well-known the predictor $\hat{X}(t, \tau)$ is given by the conditional expectation:

$$
\hat{X}(t, \tau)=E\left\{X(t+\tau) \mid \mathscr{B}_{t}(V)\right\}, \quad \mathscr{B}_{t}(V)=\sigma\{V(s) ; s \leq t\} .
$$

While A. M. Yaglom [5] has discussed the optimum mean square predictor assuming that Markov process $V(t)$ is transformed by a function $f$ with inverse $f^{-1}$ and that $X(s)=f(V(s)), s \leq t$, are observed.

In this case, it holds evidently that

$$
\begin{aligned}
\hat{X}(t, \tau) & =E\{X(t+\tau) \mid X(s) ; s \leq t\}=E\{X(t+\tau) \mid X(t)\} \\
& =E\{X(t+\tau) \mid V(t)\}=E\{X(t+\tau) \mid V(s) ; s \leq t\} .
\end{aligned}
$$

Yaglom's situation coincides with ours in the sense that the $X(s)$ are assumed to be given for $s \leq t$. In this case, too, the predictor (2) coincides with (1) actually, because $f$ is invertible.

Received May 24, 1982. 
We now clarify the best non-linear predictor of $X(t+\tau), \tau>0$, which is the main topic of this paper. Now let $\mathscr{B}_{t}(X)$ be the least $\sigma$-field generated by $X(s) ; s \leq t$, and let $\hat{X}(t, \tau)$ be the $\mathscr{B}_{t}(X)$-measurable random variable for which

$$
E|X(t+\tau)-\hat{X}(t, \tau)|^{2}
$$

attains the minimum of $E|X(t+\tau)-Y|^{2}, Y$ being $\mathscr{B}_{t}(X)$-measurable random variable with finite variance. Such a random variable $\hat{X}(t, \tau)$ always exists and it is called the best non-linear predictor of $X(t+\tau), \tau>0$. Evidently, it is given by the conditional expectation:

$$
\hat{X}(t, \tau)=E\left\{X(t+\tau) \mid \mathscr{B}_{t}(X)\right\} .
$$

It depends on the properties of the basic processes and on the structure of the function $f$ whether the explicit value of $\hat{X}(t, \tau)$ can be given or not.

We shall discuss how to obtain the best non-linear predictor $\hat{X}(t, \tau)$ of $X(t+\tau), \tau>0$, by means of the observed values $X(s), s \leq t$, here the process $\{X(s)\}$ is a new process transformed from Ornstein-Uhlenbeck process $\{U(s)\}$ by the function $f$, namely $\{X(s)\}=\{f(U(s))\}$.

As is mentioned above, the explicit form of $\hat{X}(t, \tau)$ depends on the structure of function $f$. Therefore our discussion will be restricted to the following several cases: (1)-(4). In each case we are able to obtain the exact value of the predictors.

(1) The function $f$ has a single valley (peak) and is not symmetric (cf. Theorem 1).

(2) The function $f$ has a single valley (peak) and is symmetric on a bounded interval (cf. Theorem 2).

(3) The function $f$ is symmetric on $(-\infty, \infty)$ (cf. Theorem 3).

(4) The function $f$ has several valleys (peaks) (cf. Theorem 4).

We have hopes that similar results would be obtained in more general cases where the basic processes are taken to be a multiple Markov process. Such an approach will be discussed in separate paper.

Acknowledgement. I am very grateful to Professor I. Kubo for having given me kind help for writing this paper.

\section{§1. Background}

We will take a canonical Ornstein-Uhlenbeck process $\{U(t)\}$ as the basic process, and will assume regular condition on $f$. By a canonical 
Ornstein-Uhlenbeck process we mean a Gaussian process with continuous paths, with expectation zero and with covariance $E\{U(t+\tau) U(t)\}=e^{-|\tau|}$.

The Ornstein-Uhlenbeck process $\{U(t)\}$ is not only a Gaussian stationary process but also a strong Markov process together with $\{U(-t)\}$. These properties will play important roles in the later discussion.

The semi-group $\left\{T_{\tau} ; \tau \geq 0\right\}$ of $U(t)$ is given as follows

$$
\left(T_{\imath} f\right)(x)=\int_{-\infty}^{\infty} f(y)\left[2 \pi\left(1-e^{-2 \tau}\right)\right]^{-1 / 2} \exp \left\{-\frac{\left(y-e^{-\tau} x\right)^{2}}{2\left(1-e^{-2 \tau}\right)}\right\} d y
$$

provided the integral exists.

LEMMA 1. (i ) $E\left\{X(t+\tau) \mid \mathscr{B}_{t}(U)\right\}=\left(T_{\tau} f\right)(U(t))$,

$$
\mathscr{B}_{t}(U)=\sigma\{U(s) ; s \leq t\},
$$

(ii) $\hat{X}(t, \tau)=E\left\{\left(T_{\tau} f\right)(U(t)) \mid \mathscr{B}_{t}(X)\right\}$.

Proof. (i) is clear from the Markov property of $U(t)$. (ii) is also obvious since we have (i) and

$$
\hat{X}(t, \tau)=E\left\{X(t+\tau) \mid \mathscr{B}_{t}(X)\right\}=E\left\{E\left[X(t+\tau) \mid \mathscr{B}_{t}(U)\right] \mid \mathscr{B}_{t}(X)\right\} .
$$

Our approach will be illustrated by the following three examples.

EXAMPLE 1. If $f$ is a strictly monotone function, then we see that $\mathscr{B}_{t}(U)=\mathscr{B}_{t}(X)$, since the $\sigma$-fields generated by a single random variable $U(t)$ and by $X(t)$, respectively, coincide with each other for each $t$. Therefore we easily verify

$$
\hat{X}(t, \tau)=\left(T_{\tau} f\right)(U(t))=\left(T_{\tau} f\right)\left(f^{-1}(X(t))\right) .
$$

Such cases have been discussed by Yaglom [5], Zabotina [6] and others.

ExAmple 2. Let $H_{n}(x)$ be the Hermite's polynomial of degree $n$ defined by

$$
\exp \left\{s x-\frac{1}{2} s^{2}\right\}=\sum_{n=0}^{\infty} \frac{s^{n}}{n !} H_{n}(x)
$$

and put $X(t)=H_{n}(U(t))$. If $n$ is odd, then although the $\sigma$-fields generated by $U(t)$ and $X(t)$ respectively, do not coincide with each other for each $t$, we can still show $\mathscr{B}_{t}(U)=\mathscr{B}_{t}(X)$, and hence the best non-linear predictor of $X(t+\tau)$ and the mean square error are given as follows

$$
\hat{X}(t, \tau)=e^{-n \tau} X(t), \quad \sigma^{2}(\tau)=\left(1-e^{-2 n \tau}\right) n ! .
$$


Generally, if the equality $\mathscr{B}_{t}(U)=\mathscr{B}_{t}(X)$ holds, then the best nonlinear predictor $\hat{X}(t, \tau)$ is represented by using an explicit function $\left(T_{z} f\right)(U(t))$, namely

$$
\hat{X}(t, \tau)=\left(T_{\tau} f\right)(U(t))
$$

However the value of $U(t)$ is not always determined by means of observed values $X(s)=f(U(s))$ for $s \leq t$. We are therefore interested in the cases where the value of $U(t)$ is determined from observed values under suitable conditions for $f$, so as the predictor is obtained explicitly.

EXAmple 3. We shall then discuss a case where $\mathscr{B}_{t}(U) \neq \mathscr{B}_{t}(X)$ but

$$
\hat{X}(t, \tau)=\left(T_{\tau} f\right)(U(t))
$$

does hold. For instance, let $n$ be an even number in Example 2. Then we can show that $\mathscr{B}_{t}(U) \neq \mathscr{B}_{t}(X)$ but (8) does hold, actually (7) does hold (cf. Theorem 3 and Example 5). The mean square error of the best nonlinear predictor given by (8) is

$$
\begin{array}{r}
\sigma^{2}(\tau)=\frac{1}{\sqrt{2 \pi}} \int_{-\infty}^{\infty} f(x)^{2} \exp \left(-\frac{x^{2}}{2}\right) d x-\frac{1}{2 \pi \alpha} \int_{-\infty}^{\infty} \int_{-\infty}^{\infty} f(x) f(y) \\
\times \exp \left\{-\frac{\left(x^{2}+y^{2}\right)-2 e^{-2 \tau} x y}{2 \alpha^{2}}\right\} d x d y
\end{array}
$$

with $\alpha=\left(1-e^{-4 \tau}\right)^{1 / 2}$.

The canonical Ornstein-Uhlenbeck process can be canonically represented (with respect to the past and to future, respectively) by some canonical Brownian motion $B(t)$ and $\tilde{B}(t)$ in such a way that

$$
U(t)=\sqrt{2} e^{-t} \int_{-\infty}^{t} e^{\lambda} d B(\lambda)=\sqrt{2} e^{t} \int_{t}^{\infty} e^{-\lambda} d \tilde{B}(\lambda)
$$

Here it is noted that

$$
\begin{aligned}
U(t)-U(s) & =\sqrt{2}[B(t)-B(s)]-\int_{s}^{t} U(r) d r \\
& =\sqrt{2}[\tilde{B}(s)-\tilde{B}(t)]+\int_{s}^{t} U(r) d r .
\end{aligned}
$$

Proposition 1. If the function $f$ is differentiable and $T=T(\omega)(<\infty)$ is a stopping time of $U(t)$, then

$$
O(T, \omega) \equiv \limsup _{r \downarrow T} \frac{f(U(r))-f(U(T))}{2 \sqrt{|r-T| \log \log 1 /|r-T|}}=\left|f^{\prime}(U(T))\right|
$$


holds. Moreover, if $T$ is a stopping time of the time reversed OrnsteinUhlenbeck process $\{U(-t)\}$, then also

$$
O(T, \omega) \equiv \limsup _{r \uparrow T} \frac{f(U(r))-f(U(T))}{2 \sqrt{|r-T| \log \log 1 /|r-T|}}=\left|f^{\prime}(U(T))\right| .
$$

Proof. By using the law of the iterated logarithm for Brownian motion:

$$
\limsup _{r \rightarrow s} \frac{B(r, \omega)-B(s, \omega)}{\sqrt{2|r-s| \log \log 1 /|r-s|}}=1,
$$

the formula (10) and the strong Morkov property imply the formula (11).

\section{§2. The best non-linear prediction problem for functions with single valley}

Now, we assume that the function $f$ is continuous on $(-\infty, \infty)$, strictly monotone decreasing (resp. increasing) and differentiable on $(-\infty, 0)$ (resp. on $(0, \infty)$ ) and that $f$ is normalized as $f(0)=0$.

Lemma 2. Putting $\theta=\inf \{u>0 ; f(u) \neq f(-u)\}$, we have the following:

(i) If $\theta=0$, then for any $\varepsilon>0$ there exist $u$ and $\bar{u}$ in the neighbourhood $D=\{u ;|u|<\varepsilon\}$ such that

$$
f(u)=f(\bar{u}), \quad\left|f^{\prime}(u)\right| \neq\left|f^{\prime}(\bar{u})\right|, \quad u>0, \quad \bar{u}<0 .
$$

(ii) If $0<\theta<\infty$, then for any $\varepsilon>0$ there exist $u$ and $\bar{u}$ in the neighbourhood $D=\{u ; 0<f(u)-f(\theta)<\varepsilon\}$ such that

$$
f(u)=f(\bar{u}), \quad\left|f^{\prime}(u)\right| \neq\left|f^{\prime}(\bar{u})\right|, \quad u>0, \quad \bar{u}<0 .
$$

(iii) If $\theta=\infty$, then for any $u, f(u)=f(-u)$ holds, namely the function $f$ is symmetric.

We are going to discuss our prediction problems dividing them into three cases, $\theta=0,0<\theta<\infty, \theta=\infty$, by virtue of the lemma above.

For $h>0$ the inverse image of the function $f$ consists of two points with different sign; denote by $f_{+}^{-1}(h)$ the positive one and by $f_{-}^{-1}(h)$ the negative one. Moreover, we define a stopping time $T(h, t, \omega)$ of the time reversed Ornstein-Uhlenbeck process $\{U(-t)\}$ for $h \geq 0$ by

$$
T(h) \equiv T(h, t, \omega) \equiv \sup \{q ; X(q)=h, q<t\} .
$$

If $\theta=0$, then we can choose a positive monotonical sequence $h_{n} \downarrow 0$ such that 


$$
\left|f^{\prime}\left(f_{+}^{-1}\left(h_{n}\right)\right)\right| \neq\left|f^{\prime}\left(f_{-}^{-1}\left(h_{n}\right)\right)\right|
$$

holds for each $n$, by Lemma 2 (i).

Theorem 1. If $\theta=0$, then $U(t)$ is $\mathscr{B}_{t}(X)$-measurable and the best nonlinear predictor of $X(t+\tau), \tau>0$, is given by

$$
\hat{X}(t, \tau)=\left(T_{\tau} f\right)(U(t)) .
$$

Actually, if $X(t)=0$, then the value of $U(t)$ is equal to zero. If $X(t)>0$, then taking a sequence $\left\{h_{n}\right\}$ as above and choosing $n$ with $h_{n}<X(t)$, we are given the value of $U(t)$ by

$$
U(t)= \begin{cases}f_{+}^{-1}(X(t)) & \text { if } O\left(T\left(h_{n}, t, \omega\right), \omega\right)=\left|f^{\prime}\left(f_{+}^{-1}\left(h_{n}\right)\right)\right|, \\ f_{-}^{-1}(X(t)) & \text { if } O\left(T\left(h_{n}, t, \omega\right), \omega\right)=\left|f^{\prime}\left(f_{-}^{-1}\left(h_{n}\right)\right)\right| .\end{cases}
$$

Proof of Theorem 1. We assume that for a fixed $t$ the values $\{X(r)$; $r \leq t\}$ are observed. By the conditions of this theorem and Lemma 2 (i) there exists an $h_{n}$ which satisfies (14). Let $T\left(h_{n}, t, \omega\right)$ be the stopping time defined by (13). Then by using the Proposition 1 we have

$$
O\left(T\left(h_{n}, t, \omega\right), \omega\right)=\left|f^{\prime}\left(U\left(T\left(h_{n}\right)\right)\right)\right| .
$$

Thus from (14) and (15) the values of $U\left(T\left(h_{n}\right)\right)$ are determined as follows

$$
U\left(T\left(h_{n}\right)\right)= \begin{cases}f_{+}^{-1}\left(h_{n}\right) & \text { if } O\left(T\left(h_{n}, t, \omega\right), \omega\right)=\left|f^{\prime}\left(f_{+}^{-1}\left(h_{n}\right)\right)\right|, \\ f_{-}^{-1}\left(h_{n}\right) & \text { if } O\left(T\left(h_{n}, t, \omega\right), \omega\right)=\left|f^{\prime}\left(f_{-}^{-1}\left(h_{n}\right)\right)\right| .\end{cases}
$$

Then the question is how to determine the value of $U(t)$ by means of the value $U\left(T\left(h_{n}\right)\right)$. If $X(t, \omega)>0$ and $h_{n}$ is chosen so as to hold $h_{n}<X(t, \omega)$, then by the definition of the $T\left(h_{n}, t, \omega\right), X(r)$ does not pass through the point zero in the time interval $\left(T\left(h_{n}\right), t\right)$. Hence if $U\left(T\left(h_{n}\right)\right)$ $>0(<0)$, then $U(t)>0(<0)$. Namely

$$
U(t)= \begin{cases}f_{+}^{-1}(X(t)) & \text { if } U\left(T\left(h_{n}\right)\right)=f_{+}^{-1}\left(h_{n}\right), \\ f_{-}^{-1}(X(t)) & \text { if } U\left(T\left(h_{n}\right)\right)=f_{-}^{-1}\left(h_{n}\right) .\end{cases}
$$

Therefore, the value of $U(t)$ is uniquely determined by the observed values of $X(r)$ for $r \leq t$. Hence $U(t)$ is $\mathscr{B}_{t}(X)$-measurable. Thus

$$
\hat{X}(t, \tau)=E\left\{\left(T_{\tau} f\right)(U(t)) \mid \mathscr{B}_{t}(X)\right\}=\left(T_{\tau} f\right)(U(t)) .
$$

The above results are also valid when the graph of the equation $y=f(x)$ involves parallel counter parts. 
Corollary. Suppose that the function $f$ is continuously differentiable on $(-\infty, \infty)$ except at a point $v_{0}$, strictly monotone in both sides of the point $v_{0}$ respectively and not symmetric (with respect to $v_{0}$ ) in any neighbourhood of the point $v_{0}$. Then $\mathscr{B}_{t}(X)=\mathscr{B}_{t}(U)$ holds and the best nonlinear predictor of $X(t+\tau)=f(U(t+\tau)), \tau>0$, is given by

$$
\hat{X}(t, \tau)=\left(T_{\tau} f\right)(U(t)) .
$$

Theorem 2. If $0<\theta<\infty$, then the best non-linear predictor of $X(t+\tau), \tau>0$, is given by

$$
\hat{X}(t, \tau)= \begin{cases}\left(T_{\tau} f\right)(U(t)) & \text { if } \omega \in W, \\ \frac{1}{2}\left[\left(T_{\tau} f\right)\left(f_{+}^{-1}(X(t))\right)+\left(T_{\tau} f\right)\left(f_{-}^{-1}(X(t))\right)\right] & \text { if } \omega \notin W,\end{cases}
$$

where $W \equiv\left\{\omega ; X(s, \omega)>f(\theta), T(0, t, \omega)<{ }^{3} s \leq t\right\}$ and $T(0, t, \omega)$ is given by (13). The value of $U(t, \omega)$ is given by (18) for $\omega \in W$.

Proof. Since $0<\theta<\infty$, by Lemma 2 (ii) we can choose a monotone decreasing sequence $\left\{h_{n}\right\}$ such that $h_{n} \downarrow f(\theta)$ as $n \rightarrow \infty$ and

$$
f^{\prime}\left(f_{+}^{-1}\left(h_{n}\right)\right) \neq-f^{\prime}\left(f_{-}^{-1}\left(h_{n}\right)\right)
$$

holds.

Since for any $\omega \in W$ there exists $s$ satisfying $X(s, \omega)>f(\theta), T(0, t, \omega)$ $<s \leq t$, by the continuity of the path, there exists $n$ such that

$$
T(0, t, \omega)<T\left(h_{n}, t, \omega\right) \leq t .
$$

Moreover by the Proposition 1 we have

$$
O\left(T\left(h_{n}, t, \omega\right), \omega\right)=\left|f^{\prime}\left(U\left(T\left(h_{n}\right)\right)\right)\right| \quad \text { for every } n \text {, a.e. } \omega
$$

and hence the value of $U\left(T\left(h_{n}\right)\right)$ is determined by

$$
U\left(T\left(h_{n}\right)\right)= \begin{cases}f_{+}^{-1}\left(h_{n}\right) & \text { if } O\left(T\left(h_{n}\right), \omega\right)=\left|f^{\prime}\left(f_{+}^{-1}\left(h_{n}\right)\right)\right|, \\ f_{-}^{-1}\left(h_{n}\right) & \text { if } O\left(T\left(h_{n}\right), \omega\right)=\left|f^{\prime}\left(f_{-}^{-1}\left(h_{n}\right)\right)\right| .\end{cases}
$$

Furthermore by using the arguments similar to the proof of Theorem 1 , we obtain

$$
U(t)=\left\{\begin{array}{lll}
f_{+}^{-1}(X(t)) & \text { if } O\left(T\left(h_{n}\right), \omega\right)=\left|f^{\prime}\left(f_{+}^{-1}\left(h_{n}\right)\right)\right|, \quad \omega \in W, \\
f_{-}^{-1}(X(t)) & \text { if } O\left(T\left(h_{n}\right), \omega\right)=\left|f^{\prime}\left(f_{-}^{-1}\left(h_{n}\right)\right)\right|, \quad \omega \in W .
\end{array}\right.
$$

Therefore the value of $U(t)$ is uniquely determined in terms of the observed values $X(r)$ for $r \leq t$ under the condition $W$ that there exists 
$s$ such that $X(s)>f(\theta)$ and $T(0, t, \omega)<s \leq t$. Namely under this condition $U(t)$ is $\mathscr{B}_{t}(X)$-measurable. However, since

$$
\begin{aligned}
\hat{X}(t, \tau) & =E\left\{\left(T_{\tau} f\right)(U(t)) \mid \mathscr{B}_{t}(X)\right\} \\
& =\left(T_{\tau} f\right)(U(t)) \chi_{W}+\chi_{W c} E\left\{\left(T_{\tau} f\right)(U(t)) \mid \mathscr{B}_{t}(X)\right\},
\end{aligned}
$$

we have

$$
\hat{X}(t, \tau)=\left(T_{\imath} f\right)(U(t)), \quad \omega \in W .
$$

If $\omega \notin W$, i.e. $X(s, \omega) \leq f(\theta)$ for any $s$ in the time interval $(T(0, t, \omega), t]$, then during $(T(0, t, \omega), t], U(s)$ stays within the interval $[-\theta, \theta]$, on which the function $f$ is symmetric with respect to the axis of the ordinate. Although the value of $U(s)$ is not uniquely determined by the value of $X(s)$, the best non-linear predictor of $X(t+\tau), \tau>0$, under the condition $W^{c}$ is given by

$$
\begin{aligned}
\hat{X}(t, \tau) & =E\left\{\left(T_{\tau} f\right)(U(t)) \mid \mathscr{B}_{t}(X)\right\} \\
& =E\left\{\left(T_{\tau} f\right)(U(t)) \chi_{W c} \mid \mathscr{B}_{t}(X)\right\} \quad \text { for } \omega \notin W .
\end{aligned}
$$

To complete the proof of the theorem it is sufficient to show that

$$
E\left\{\left(T_{\tau} f\right)(U(t)) \chi_{W^{c}} \mid \mathscr{B}_{t}(X)\right\}=\frac{1}{2}\left\{\left(T_{\tau} f\right)(|U(t)|)+\left(T_{\tau} f\right)(-|U(t)|)\right\} \chi_{W^{c}} .
$$

Since $W \in \mathscr{B}_{t}(X)$, the $\chi_{W c}(\omega)$ is $\mathscr{B}_{t}(X)$-measurable so is the right-hand side of (21). Therefore for any $G \in \mathscr{B}_{t}(X)$, we must show

$$
\begin{aligned}
& \int_{G}\left(T_{\tau} f\right)(U(t)) \chi_{W c} d P(\omega) \\
& \quad=\int_{G} \frac{1}{2}\left\{\left(T_{\tau} f\right)(|U(t)|)+\left(T_{\tau} f\right)(-|U(t)|)\right\} \chi_{W^{c}} d P(\omega) .
\end{aligned}
$$

It can be shown by noting the strong Markov property of $U(t)$, the symmetry of the probability measure of Ornstein-Uhlenbeck process starting from the origin.

Theorem 3. If $\theta=\infty$, then the best non-linear predictor of $X(t+\tau)$, $\tau>0$, is given by

$$
\hat{X}(t, \tau)=\left(T_{\tau} f\right)(U(t))=\left(T_{\tau} f\right)\left(f_{+}^{-1}(X(t))\right)=\left(T_{\tau} f\right)\left(f_{-}^{-1}(X(t))\right) .
$$

Proof. Since $f(u)=f(-u)$ holds for any $u$, it is easy to verify

$$
\left(T_{\imath} f\right)(u)=\left(T_{\tau} f\right)(-u)=\left(T_{\imath} f\right)(|u|) .
$$

Hence we have 


$$
\begin{aligned}
\hat{X}(t, \tau) & =E\left\{\left(T_{\tau} f\right)(U(t)) \mid \mathscr{B}_{t}(X)\right\}=E\left\{\left(T_{\tau} f\right)(U(t)) \mid f(U(s)) ; s \leq t\right\} \\
& =E\left\{\left(T_{\imath} f\right)(|U(t)|)|| U(s) \mid ; s \leq t\right\}=\left(T_{\tau} f\right)(|U(t)|) \\
& =\left(T_{\tau} f\right)(-|U(t)|)=\left(T_{\tau} f\right)\left(f_{+}^{-1}(X(t))\right)=\left(T_{\tau} f\right)\left(f_{-}^{-1}(X(t))\right) .
\end{aligned}
$$

ExAmple 4. Set

$$
f(u)= \begin{cases}-a u & \text { if } u<0, \\ b u & \text { if } u \geq 0\end{cases}
$$

with $a \neq b, a b>0$, and put $X(t)=f(U(t))$. Then we can easily have $\theta=0$, and hence we obtain

$$
\begin{aligned}
\hat{X}(t, \tau)= & \left(T_{\imath} f\right)(U(t))=\frac{(a+b) \delta}{\sqrt{2 \pi}} \exp \left\{-\frac{e^{-2 \tau}}{2 \delta^{2}} U(t)^{2}\right\} \\
& +(b-a) e^{-\tau} U(t)\left\{\Phi\left(\frac{e^{-\tau}}{\delta} U(t)\right)-\Phi\left(-\frac{e^{-\tau}}{\delta} U(t)\right)\right\}
\end{aligned}
$$

where

$$
\Phi(r)=\frac{1}{\sqrt{2 \pi}} \int_{-\infty}^{r} \exp \left(-\frac{y^{2}}{2}\right) d y \quad \text { and } \quad \delta=\sqrt{1-e^{-2 \tau}}
$$

Example 5. Set $X(t)=f(U(t))=\beta|U(t)|, \beta \neq 0$. Then we obtain

$$
\begin{aligned}
\hat{X}(t, \tau)= & \left(T_{\imath} f\right)(U(t))=\sqrt{\frac{2}{\pi}} \beta \delta \exp \left\{-\frac{1}{2} e^{-2 \tau} \beta^{-2} \delta^{-2} X(t)^{2}\right\} \\
& +\beta e^{-\tau} U(t)\left\{\Phi\left(\frac{e^{-\tau}}{\delta} U(t)\right)-\Phi\left(-\frac{e^{-\tau}}{\delta} U(t)\right)\right\} .
\end{aligned}
$$

ExAmple 6. Set $X(t)=f(U(t))=(U(t))^{n}, n=1,2, \cdots$. Then we obtain

$$
\hat{X}(t, \tau)=\left(T_{\imath} f\right)(U(t))=\sum_{\ell=0}^{[n / 2]}\left(\begin{array}{c}
n \\
2 \ell
\end{array}\right) \exp \{-\tau(n-2 \ell)\} \delta^{2 \ell}(2 \ell-1) ! ! X(t)^{(n-\Omega \ell) / n} .
$$

\section{$\S 3$. The best non-linear prediction problem with several peaks}

We have discussed the prediction problem for a simpler function $f$ in Section 2. We now extend Theorem 1 for more general function $f$. Assume that the function $f$ is continuously differentiable on $(-\infty, \infty)$ except at finite points $\left\{\alpha_{1}, \alpha_{2}, \cdots, \alpha_{n}\right\}$ and that $f$ is strictly monotone on each interval $\left(\alpha_{k}, \alpha_{k+1}\right), \kappa=0,1,2, \cdots, n$, where $\alpha_{0}=-\infty, \alpha_{n+1}=\infty$. Moreover assume that the function $f$ is not symmetric in any neighbourhood of the points $\alpha_{k}$, namely for any $\varepsilon>0$ there exist $u_{\kappa 1}$ and $u_{\kappa 2}$ in the neighbourhood $D_{\kappa}=\left\{u ;\left|u-\alpha_{\kappa}\right|<\varepsilon\right\}, \kappa=1,2, \cdots, n$, such that 


$$
f\left(u_{\kappa 1}\right)=f\left(u_{\kappa 2}\right), \quad\left|f^{\prime}\left(u_{\kappa 1}\right)\right| \neq\left|f^{\prime}\left(u_{\kappa 2}\right)\right|, \quad u_{\kappa 1}<\alpha_{\kappa}<u_{\kappa 2} .
$$

As in Section 2, under these conditions, the predictor is given by

$$
\hat{X}(t, \tau)=\left(T_{\imath} f\right)(U(t)),
$$

and the algorithm to determine the value of $U(t)$ is seen.

For simplicity we shall consider only the case where the function $f$ possesses one maximal value and one minimal value: $f_{\text {maximal }}=f\left(\alpha_{1}\right), f_{\text {minimal }}$ $=f\left(\alpha_{2}\right)$. Divide the region $(-\infty, \infty)$ of $u$ into three intervals: $I_{1}=$ $\left(-\infty, \alpha_{1}\right], I_{2}=\left(\alpha_{1}, \alpha_{2}\right]$ and $I_{3}=\left(\alpha_{2}, \infty\right)$. Denote by $f_{j}$ the restriction of $f$ to $I_{j}$ and define $f_{j}^{-1}$ on the interval $I_{j}$. Then of course, $-\infty=\alpha_{0}<f_{1}^{-1}(x)$ $\leq \alpha_{1} \leq f_{2}^{-1}(x) \leq \alpha_{2} \leq f_{3}^{-}(x)<\alpha_{3}=\infty$.

We suppose that $X(r), r \leq t$, are observed. Once we know the interval $I_{j}$ to which $U(r)$ belongs at a given time $r(\leq t)$, then we can immediately determine the value of $U(r)$ by the value of $X(r)$ in such a way that

$$
U(r)=f_{j}^{-1}(X(r)) .
$$

In view of this we will first show that there exists at least one random time point $t_{0}(<t)$ such that the interval $I_{j}$ including $U\left(t_{0}\right)$ is determined at the time. Secondly, we will show that we can trace the intervals which include $U(s)$ after the time $t_{0}$ by observing $X(s), t_{0} \leq s$ $\leq t$.

By the property of the function $f$ there exist $h^{*}$ and $j_{0}$ such that

$$
\left|f^{\prime}\left(f_{j}^{-1}\left(h^{*}\right)\right)\right| \neq\left|f^{\prime}\left(f_{j_{0}}^{-1}\left(h^{*}\right)\right)\right|, \quad j \neq j_{0} .
$$

For instance, if either $f\left(\alpha_{2}\right)>f\left(\alpha_{0}\right) \equiv \lim _{u \rightarrow-\infty} f(u)$ or $f\left(\alpha_{1}\right)<f\left(\alpha_{3}\right) \equiv$ $\lim _{u \rightarrow \infty} f(u)$ holds, then we may take $h^{*}$ in such way that

$$
f\left(\alpha_{2}\right)>h^{*}>f\left(\alpha_{0}\right) \text { or } f\left(\alpha_{1}\right)<h^{*}<f\left(\alpha_{3}\right) .
$$

Even in the contrary case we can choose $h^{*}$ which satisfies the above condition.

For the $h^{*}$ define a stopping time $T\left(h^{*}, t, \omega\right)$ by

$$
T\left(h^{*}\right) \equiv T\left(h^{*}, t, \omega\right)=\sup \left\{q ; X(q)=h^{*}, q<t\right\},
$$

then by the property of Ornstein-Uhlenbeck process we can easily see that $T\left(h^{*}, t, \omega\right)>-\infty$. Therefore by using the argument similar to that in the proof of the Theorem 1 we can determine the value of $U\left(T\left(h^{*}\right)\right)$, 
in particular we know the interval which includes $U\left(T\left(h^{*}\right)\right)$.

Suppose that at a given time $r(<t)$ the interval including $U(r)$ is known, say $I_{j}$. If the value $X(s)$ hits $f\left(\alpha_{j}\right)$ at a time earlier than $f\left(\alpha_{j-1}\right)$ after the time $r$, then we can know which $I_{j}$ or $I_{j+1}$ does include $U(s)$, for $s$ in the time interval between $r$ and the first hitting time to the set $\left\{f\left(\alpha_{j-1}\right), f\left(\alpha_{j+1}\right)\right\}$ after the above hitting time to $f\left(\alpha_{j}\right)$, by a similar way to the proof of Theorem 1 observing the variations of $X$ at points $h$ such that (23) holds with $h=f\left(u_{j 1}\right)=f\left(u_{j 2}\right)$. Namely, the value of $U(s)$ is determined for any $s$ in the above time interval. In the complementary case, the value of $U(s)$ is similarly determined for any $s$ in the time interval between $r$ and the first hitting time to $\left\{f\left(\alpha_{j-2}\right), f\left(\alpha_{j}\right)\right\}$ after the first hitting time to $f\left(\alpha_{j-1}\right)$ after $r$.

We have thus known the value of $U(r)$ at the time $r=T\left(h^{*}\right)$. Then applying the above discussion recursively, we can determine the value of $U(s), T\left(h^{*}\right) \leq s \leq t$, especially the value of $U(t)$, in terms of $X(s), s \leq t$. Thus we see that $U(t)$ is $\mathscr{B}_{t}(X)$-measurable, and hence we have proved the following theorem.

THEOREM 4. If the function $f$ satisfies the condition explained in the $b$ eginning of this section. Then for $X(t)=f(U(t))$ the equality $\mathscr{B}_{t}(X)=$ $\mathscr{B}_{t}(U)$ valid is and the best non-linear predictor of $X(t+\tau), \tau>0$ is given by

$$
\hat{X}(t, \tau)=\left(T_{\tau} f\right)(U(t))
$$

\section{REFERENCES}

[1] I. I. Gihman and A. V. Skorohod, The Theory of Stochastic Processes, SpringerVerlag, Berlin, 1974.

[2] Yu. A. Rozanov, Stationary random processes, Holden-Day, San Francisco, 1967.

[ 3 ] T. Hida and G. Kallianpur, The square of a Gaussian Markov process anu nonlinear prediction, Multivar. Anal., 5 No. 4 (1975), 451-461.

[4] A. V. Balakrishnan, Prediction theory for Markoff processes, Pacific J. Math., 11 (1961), 1171-1182.

[ 5 ] А. М. Яглом, Примеры оптимального нелинейного зкстраполирования стационарных случайных процессов. Tp. VI Всес. совещ. по теории вероятностей и матем. статистике. Вильнюс-Паланага, 1960, Вильнюс, Гос. изд-во полит. и научн. лиа-ры ЛитССР, (1962), 275-296.

[ 6 ] Н.П. Заботина, Онтимальных нелинейные зкстраполяция, Фильтрация и интерполяция Функций от Гауссовских процессов. Проблемы передачи информаций, Том XI, Вып. 2, (1975), 84-95. 
Department of Mathematics

Faculty of Science

Nagoya University

Japan

Current Address:

Shenyang Chemical Engineering Institute

Shenyang

China 